\title{
Pulsatile ocular blood flow study: decreases in exudative age related macular degeneration
}

Fumihiko Mori, Suguru Konno, Taiichi Hikichi, Yuji Yamaguchi, Satoshi Ishiko, Akitoshi Yoshida
Department of Ophthalmology, Asahikawa Medical College, Asahikawa, Japan

F Mori

S Konno

T Hikichi

Y Yamaguchi

S Ishiko

A Yoshida

Correspondence to: Fumihiko Mori, MD, PhD, Department of Ophthalmology, Asahikawa Medical College, Midorigaoka Higashi 2-1-1-1, Asahikawa 078-8510, Japan morinaod5.dion.ne.jp

Accepted for publication 13 February 2001

\begin{abstract}
Background-Pulsatile ocular blood flow (POBF) is a parameter for evaluating choroidal blood flow. POBF in the patients with non-exudative and exudative age related macular degeneration (AMD) was investigated.

Methods-POBF, pulse amplitude (PA), systolic and diastolic blood pressures, intraocular pressure (IOP), refractive error, and axial length were compared among 10 patients with non-exudative AMD, 11 patients with exudative AMD, and 69 age matched controls. A Langham OBF computerised tonometer was used with the participants in the sitting position to measure POBF and PA.

Results-No significant differences were found in age, systolic and diastolic blood pressures, IOP, or refractive error between patients with exudative and nonexudative AMD and the control subjects. In the patients with exudative AMD the POBF (median, $372.7 \mu 1 / \mathrm{min}$ ) and PA (median, $1.2 \mathrm{~mm} \mathrm{Hg}$ ) were significantly lower than in the patients with nonexudative AMD (median, 607.0 $\mu 1 / \mathrm{min}(p=$ $0.02)$ and $2.2 \mathrm{~mm} \mathrm{Hg}(\mathrm{p}=0.04)$, respectively) and control subjects (median, 547.4 $\mu 1 / \mathrm{min}(\mathrm{p}=0.01)$ and $2.0 \mathrm{~mm} \mathrm{Hg}(\mathrm{p}=$ 0.01 ), respectively).

Conclusions-These data show that the POBF and PA in the patients with exudative AMD are lower than in the patients with non-exudative AMD and normal subjects. Decreased choroidal blood flow may have a role in the development of choroidal neovascularisation in AMD.

(Br f Ophthalmol 2001;85:531-533)
\end{abstract}

Age related macular degeneration (AMD) is the major cause of irreversible blindness in elderly patients worldwide. ${ }^{1-4}$ The pathogenesis of this disease has been investigated from genetic, ${ }^{5}$ histological, ${ }^{6}$ and haemodynamic perspectives, ${ }^{7-10}$ but it is poorly understood. AMD is classified as non-exudative, the dry type, or exudative neovascular, the wet type..$^{11}$ Choroidal neovascularisation (CNV) in the macula causes severe visual impairment in AMD. Vascular endothelial growth factor (VEGF) and other angiogenetic factors, which are induced by hypoxia and ischaemia, may play a part in the development of $\mathrm{CNV}^{13-15}$ Choroidal circulation may be important for the development of CNV, the late stage of AMD.

Several authors have evaluated ocular blood flow in patients with AMD. ${ }^{7-10}$ However, the choroidal blood flow is uncertain in patients with non-exudative and exudative AMD. Determining pulse amplitude (PA) and pulsatile ocular blood flow (POBF) by measuring intraocular pressure (IOP) using the Langham $\mathrm{OBF}$ computerised tonometer is a way to evaluate choroidal blood flow. ${ }^{16-18}$ In the current study, we evaluated the total choroidal blood flow in the patients with non-exudative and exudative AMD by measuring POBF.

\section{Materials and methods}

Patients and control subjects from the department of ophthalmology of Asahikawa Medical College were enrolled in this study. Only one eye of each participant was included. The eyes were divided as follows into three groups based on the findings of slit lamp biomicroscopy, indirect ophthalmoscopy, fundus photography, and fluorescein angiography: 69 age matched controls, 10 patients with non-exudative AMD, and 11 patients with exudative AMD. The exudative and non-exudative forms of AMD have been defined previously. ${ }^{11}{ }^{12}$ Subjects with any other ocular disease were excluded. Subjects with systemic hypertension were included in this study.

Informed consent was obtained in all cases. The study protocol was reviewed by the ethics committee of our institution. All procedures adhered to the tenets of the Declaration of Helsinki.

POBF and PA were measured by the Langham OBF computerised tonometer (Langham Ophthalmic Technologies, Timonium) with patients in the sitting position. Data were collected for POBF and PA for each subject, and the mean measurements were calculated from five representative pulses. Systolic and diastolic brachial arterial blood pressures were measured with a sphygmomanometer. IOP was measured with a non-contact tonometer (CT-90, Topcon, Japan). The refractive error was expressed as a spherical equivalent refraction obtained by autokeratorefractometer (KR-7100, Topcon). Axial length was measured using an A-scan biometric ultrasound, OcuScan (Alcon, Fort Worth).

POBF, PA, age, systolic and diastolic blood pressures, IOP, refractive error, and axial length were compared in the three groups. All comparisons were performed using the MannWhitney test. A p value $<0.05$ was considered statistically significant.

\section{Results}

No significant differences were found in age, systolic and diastolic blood pressures, IOP, refractive error, and axial length between 
Table 1 Characteristics of controls and patients with age related macular degeneration (AMD)

\begin{tabular}{llll}
\hline & Controls $(n=69)$ & Non-exudative AMD $(n=10)$ & Exudative AMD (n=11) \\
\hline Age (years) & $71(60-84)$ & $68(62-80)$ & $74(61-85)$ \\
Systolic blood pressure (mm Hg) & $140(110-200)$ & $132(120-156)$ & $140(112-172)$ \\
Diastolic blood pressure (mm Hg) & $80(60-100)$ & $78(68-98)$ & $78(64-94)$ \\
Intraocular pressure (mm Hg) & $14(9-20)$ & $15(6-20)$ & $12(10-18)$ \\
Refractive error (dioptres) & $0.5(-5.0$ to +4.6$)$ & $-0.1(-1.8$ to +4.6$)$ & $-0.3(-2.8$ to +2$)$ \\
Axial length (mm) & $23.0(20.6-25.5)$ & $22.9(20.1-24.5)$ & $23.1(21.9-23.6)$ \\
\hline
\end{tabular}

patients with AMD and the control subjects (Table 1). In the patients with exudative AMD, the PA (median $1.2 \mathrm{~mm} \mathrm{Hg}$; range 0.92.1) was significantly lower than in patients with non-exudative AMD (median $2.2 \mathrm{~mm}$ $\mathrm{Hg}$; range $0.9-3.7 ; \mathrm{p}=0.04$ ) and age matched controls (median $2.0 \mathrm{~mm} \mathrm{Hg}$; range $0.7-4.6$; $\mathrm{p}$ $=0.01$ ) (Fig 1). In patients with exudative $\mathrm{AMD}$, the POBF (median $372.7 \mu \mathrm{l} / \mathrm{min}$; range 261.6-570.2) was significantly lower than in patients with non-exudative AMD (median $607.0 \mu \mathrm{l} / \mathrm{min}$; range $401.0-959.0 ; \mathrm{p}=0.02$ ) and age matched controls (median 547.4 $\mu \mathrm{l} / \mathrm{min}$; range $290.7-1282.9 ; \mathrm{p}=0.01$ ) (Fig 2). No significant differences were found in PA and POBF between patients with nonexudative AMD and the age matched controls ( $\mathrm{p}=0.96$ and $\mathrm{p}=0.69$, respectively) (Figs 1 and 2).

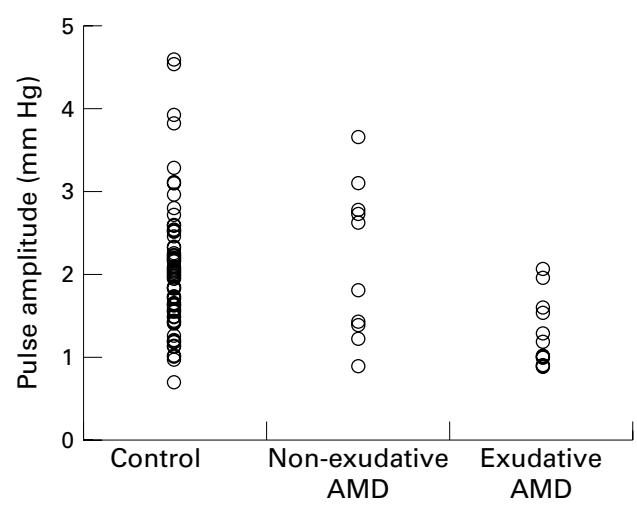

Figure 1 Pulse amplitude in patients with exudative age related macular degeneration $(A M D)(n=11)$, patients with non-exudative $A M D(n=10)$, and age matched controls $(n=69)$.

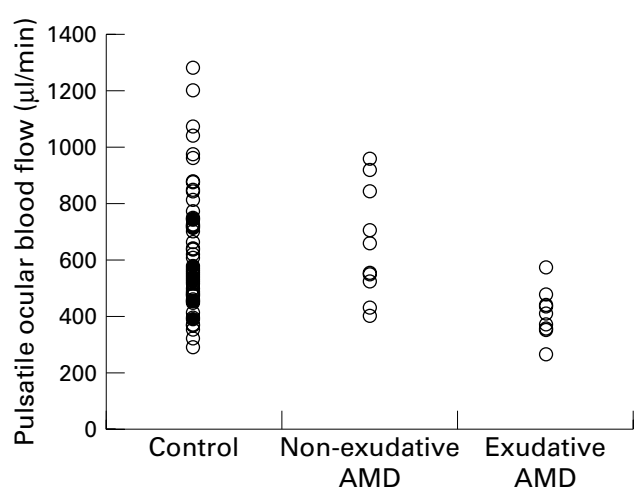

Figure 2 Pulsatile ocular blood flow in patients with exudative age related macular degeneration (AMD) $(n=11)$, patients with non-exudative $A M D(n=10)$, and age matched controls $(n=69)$.

\section{Discussion}

This study demonstrates that the PA and POBF in patients with exudative AMD are significantly lower than in controls. Several authors reported that the blood velocities of the short posterior ciliary artery are lower in the patients with AMD. ${ }^{8}$ These results indicate that the total choroidal blood flow is lower in the patients with exudative AMD than in controls. A prolonged choroidal filling phase on fluorescein angiography is related to thickening of the Bruch's membrane. ${ }^{19}$ The thickening of the Bruch's membrane may induce increased vascular resistance of the choroid and decreased choroidal blood flow in the patients with AMD.

Grunwald et al reported that the choroidal blood flow in the centre of the fovea was lower in the patients with non-exudative AMD than in age matched controls, primarily because of decreased blood volume measured by laser Doppler flowmetry. ${ }^{7}$ However, our results showed that POBF did not decrease in the patients with non-exudative AMD compared with age matched controls. Thus, our results suggest that only local choroidal blood flow in the centre of the fovea may decrease, but total choroidal blood flow does not decrease in the patients with non-exudative AMD compared with age matched controls.

Friedman et al reported that scleral rigidity increases and the choroidal blood flow decreases in the patients with AMD. ${ }^{9-21}$ Those authors concluded that the eyes of patients with AMD had decreased choroidal blood flow and increased resistance in the choroidal vasculature. Several investigators reported that scleral rigidity affects POBF. ${ }^{22}{ }^{23}$ We believe that we need to consider the relation between scleral rigidity and $\mathrm{POBF}$ in the patients with AMD in the future.

Lang et al showed that the POBF decreased in the patients with non-exudative AMD compared with controls. ${ }^{10}$ In our data, axial length was a major factor affecting POBF in normal subjects. $^{24}$ When evaluating the choroidal blood flow by measuring POBF, the effect of axial length should be considered. In the present study, no significant differences were found in the axial length in any group. We concluded that the total choroidal blood flow in patients with exudative AMD was lower than the patients with non-exudative AMD and age matched controls.

$\mathrm{CNV}$ in the macula causes severe visual impairment in the late stage of AMD. Basic fibroblast growth factor (bFGF) has been detected in CNV membranes in the patients with AMD. ${ }^{15}$ In an experimental model of 
$\mathrm{CNV}$, investigators observed a defect in Bruch's membrane and increased expression of VEGF in the accumulating macrophages and migrating retinal pigment epithelial cells and Müller cells. ${ }^{14}$ In the vitreous of patients with $\mathrm{CNV}$, the levels of VEGF increased. ${ }^{25}$ Adamis et al indicated that retinal pigment epithelial cells can promote endothelial proliferation via the production and release of VEGF during hypoxia. ${ }^{26}$ These reports suggest that angiogenic factors may play a part in the formation of CNV. In the present study, POBF in patients with AMD decreased by about $30 \%$ compared with controls and patients with non-exudative AMD. The decreased total choroidal blood flow may induce $\mathrm{CNV}$ via angiogenic factor induced by hypoxia in the patients with exudative AMD. Further studies of choroidal circulation, which may be important for the development of $\mathrm{CNV}$ in the late stage of $\mathrm{AMD}$, are needed.

The authors have no proprietary interest in any aspect of this technology.

1 Ghafour IM, Allan D, Foulds WS. Common cause of blindness and visual handicap in the west of Scotland. $\mathrm{BrF} \mathrm{Oph}$ thalmol 1983;67:209-13.

2 Attebo K, Mitchell P, Smith W. Visual acuity and the causes of visual loss in Australia: the Blue Mountains Eye Study. Ophthalmology 1996;103:357-64.

3 Klein R, Wang Q, Klein BEK, et al. The relationship of agerelated maculopathy, cataract, and glaucoma to visual acuity. Invest Ophthalmol Vis Sci 1995;36:182-91.

4 Klein R, Klein BEK, Lee KE. Changes in visual acuity in a population: the Beaver Dam Eye Study. Ophthalmology 1996;103:1169-78.

5 Allikmets R, Shroyer N, Singh N, et al. Mutation of the Stargardt disease gene (ABCR) in age-related macular degeneration. Science 1997;277:1805-7.

6 Young R. Pathophysiology of age-related macular degeneration. Surv Ophthalmol 1987;31:291-306.

7 Grunwald JE, Hariprasad SM, Dupont J, et al. Foveal choroidal blood flow in age-related macular degeneration. Invest Ophthalmol Vis Sci 1998;39:385-90.

8 Ciulla TA, Harris A, Chung HS, et al. Color Doppler imaging discloses reduced ocular blood flow velocities in nonexudative age-related macular degeneration. $A m \AA$ Ophthalmol 1999;128:75-80.
9 Friedman E, Krupsky S, Lane AM, et al. Ocular blood flow velocity in age-related macular degeneration. Ophthalmolvelocity in age-relate
ogy 1995;102:640-6.

10 Lang GE, Peisert T, Hogel J, et al. Ocular blood flow in agerelated macular degeneration. Ophthalmology 1994;101 (suppl): 141

11 Sarks SH, Sarks JP. Age-related macular degeneration: atropic form. In: Schachat AP, Murphy RP, eds. Retina. 2nd ed. St Louis: Mosby, 1994;Vol 2:67.

12 Elman MJ, Fine SL. Exudative age-related macular degeneration. In: Schachat AP, Murphy RP, eds. Retina. 2nd ed. St Louis: Mosby, 1994; Vol 2:68.

13 Shweiki D, Itin A, Soffer D, et al. Vascular endothelial growth factor induced by hypoxia may mediate hypoxiainitiated angiogenesis. Nature 1992;359:843-5

14 Ishibashi T, Hata Y, Yoshikawa H, et al. Expression of vascular endothelial growth factor in experimental choroidal neovascularization. Graefes Arch Clin Exp Ophthalmol 1997; 235:159-67.

15 Amin R, Puklin JE, Frank RN. Growth factor localization in choroidal neovascular membranes of age-related macular degeneration. Invest Ophthalmol Vis Sci 1994;35:3178-88.

16 Langham ME, Farrell RA, O'Brien V, et al. Noninvasive measurement of pulsatile blood flow in the human eye. In: Lambrou GN, Greve EL, eds. Ocular blood flow in glaucoma. Amsterdam: Kugler and Ghedini, 1989.

17 Alm A, Bill A. Ocular circulation. In: Moses R, Hat W, eds. Adler's physiology of the eye. 9th ed. St Louis: Mosby, 1992:6.

18 Spraul CW, Lang GE, Ronzani M, et al. Reproducibility of measurements with a new slit lamp-mounted ocular blood flow tonograph. Graefes Arch Clin Exp Ophthalmol 1998; 236:274-9.

19 Pauleihoff D, Chen JC, Chisholm, IH, et al. Choroidal perfusion abnormality with age-related Bruch's membrane change. Am f Ophthalmol 1990;109:211-7.

20 Friedman E, Ivry M, Ebert E, et al. Increased scleral rigidity and age-related macular degeneration. Ophthalmology 1989;96:104-8.

21 Friedman E. The role of athrosclerotic process in the pathogenesis of age-related macular degeneration. $\mathrm{Am} \mathcal{F}$ Ophthalmol 2000;130: 658-63.

22 James CB, Trew DR, Clark K, et al. Factors influencing the ocular pulse-axial length. Graefes Arch Clin Exp Ophthalmol 1991;229:341-4.

23 Ravalico G, Pastori G, Croce M, et al. Pulsatile ocular blood flow variations with axial length and refractive error. Ophthalmologica 1997;211:271-3.

24 Mori F, Konno S, Hikichi T, et al. Factors affecting pulsatile ocular blood flow in normal subjects. $\mathrm{Br} \mathcal{F}$ Ophthalmol 2001;85:529-30.

25 Wells JA, Murthy R, Chibber R, et al. Levels of vascular endothelial growth factor are elevated in the vitreous of patients with subretinal neovascularization. $\mathrm{Br} \mathcal{F}$ Ophthalmol 1996;80:363-6.

26 Adamis AP, Shima DT, Yeo K-T, et al. Synthesis and secretion of vascular permeability factor/vascular endothelial growth factor by human retinal pigment epithelial cells. Browth factor by human retinal pigment epith 